
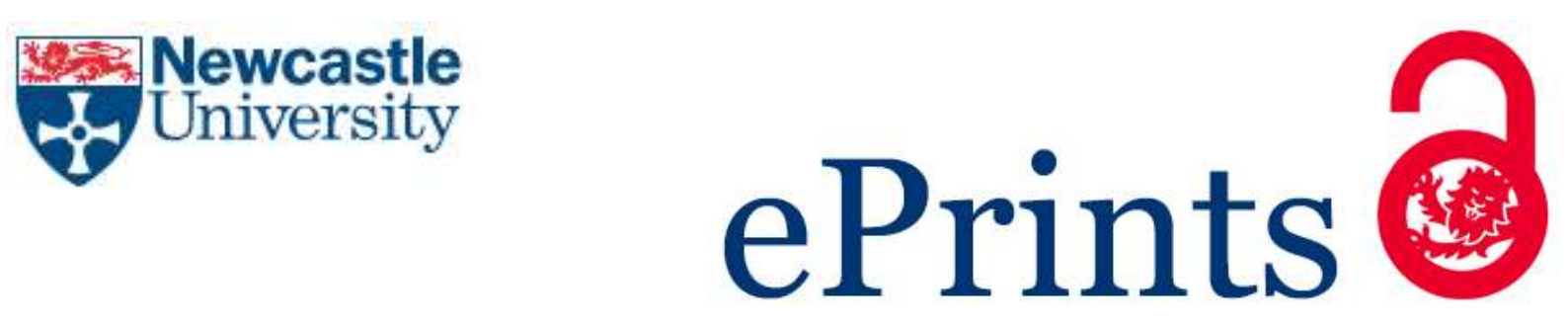

Thomas M, Robertson N, Miller N, Rankin J, McKean M, Brodlie M.

Congenital lung agenesis: incidence and outcome in the North of England.

Birth Defects Research Part A: Clinical \& Molecular Teratology 2017, 109(11), 857-859.

\title{
Copyright:
}

This is the peer reviewed version of the following article: Thomas M, Robertson N, Miller N, Rankin J, McKean M, Brodlie M. Congenital lung agenesis: incidence and outcome in the North of England. Birth Defects Research Part A: Clinical \& Molecular Teratology 2017, 109(11), 857-859., which has been published in final form at https://doi.org/10.1002/bdr2.1011 This article may be used for non-commercial purposes in accordance with Wiley Terms and Conditions for Self-Archiving.

Date deposited:

04/07/2017

Embargo release date:

12 April 2018

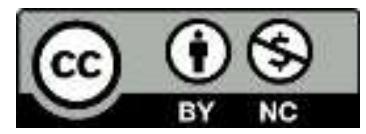

This work is licensed under a Creative Commons Attribution-NonCommercial 3.0 Unported License 


\section{Congenital lung agenesis: incidence and outcome in the North of England}

\section{Corresponding author:}

Dr Matthew Thomas

Address: $\quad$ Great North Children's Hospital

Royal Victoria Infirmary

Victoria Road

Newcastle upon Tyne

UK

NE1 4LP

Email: $\quad$ matthew.thomas3@ncl.ac.uk

Telephone: 01912821720

\section{Co-authors:}

Nic Robertson, Neonatal Intensive Care Unit, The James Cook University Hospital, Middlesbrough, UK

Nicola Miller, Regional Maternity Survey Office, Public Health England, Newcastle upon Tyne, UK

Judith Rankin, Institute of Health \& Society, Newcastle University, Newcastle upon Tyne, UK

Michael McKean, Great North Children's Hospital, Royal Victoria Infirmary, Newcastle upon Tyne, UK

Malcom Brodlie, Great North Children's Hospital, Royal Victoria Infirmary, Newcastle upon Tyne, UK and Institute of Cellular Medicine, Newcastle University, UK.

Key words: lung agensis, pulmonary aplasia, congenital anomaly

Word count: 1200 words 


\title{
Congenital lung agenesis: incidence and outcome in the North of England
}

\begin{abstract}
Unilateral absence of lung tissue and bronchial tree is an uncommon congenital abnormality. Previous reports have estimated an incidence of 1 in 15,000 pregnancies but accurate data has not been available. Here, we use the North of England register of congenital anomalies (NorCAS) to calculate the incidence as 0.12 per 100,000 live births (95\% confidence interval 0.03-0.31). We briefly report the course of the four patients with this condition born in the region between 2004 and 2013. Contrary to previous reports, the medium term outcomes in our patients have been good, even when lung agenesis is associated with other congenital anomalies such as congenital heart disease. Long-term prognosis with modern management remains unknown.
\end{abstract}

\section{INTRODUCTION}

Lung agenesis is a rare congenital anomaly in which failure of the embryological lung bud to develop results in unilateral absence of lung tissue and the bronchial tree. [1] Recent literature has quoted an estimated incidence of 1 in 15,000 pregnancies but accurate incidence data has not previously been available. [2]

Prognosis is also uncertain but is generally reported as poor in older literature, especially when lung agenesis is associated with anomalies in other organs. [2] A recent systematic review identified a total of 96 cases of lung agenesis reported in the years 1993 to 2013. [3] All 23 cases where this was an isolated anomaly were alive at the time of reporting apart from one case which underwent termination of pregnancy. Outcomes in the 73 cases associated with other anomalies were more variable: just under half (35 patients) were alive at the time of reporting, with 27 reported to have died, 2 terminations of pregnancy and 9 cases where outcome was not reported. The most common other anomaly was congenital heart disease but there was a wide-range of associations including tracheal, skeletal, renal, gastrointestinal and facial defects.

The North of England has a registry of congenital anomalies - the Northern Congenital Abnormality Survery (NorCAS) - which routinely collects prospective data on all congenital malformations. [4] There is also a centralised tertiary respiratory paediatric service which manages all confirmed cases of lung agenesis. We were therefore able to identify and follow-up all cases in order to more accurately report the incidence and contemporary outcomes of this rare condition.

\section{METHODS}

The NorCAS database records all cases of congenital anomalies detected from prenatal diagnosis to age 12 in the population of the North of England, as previously described. [4] The database was interrogated to find cases of pulmonary agenesis between 2004 and 2013 
together with figures for all live births. Case notes were reviewed. Parents or carers of all patients provided signed consent for publication of anonymous case summaries. Confidence intervals for Poisson rates were calculated using the EpiTools package for R.

\section{RESULTS}

\section{Incidence}

Between the years 2004 to 2013 there were 328,842 live born infants in the North of England and 4 infants born with lung agenesis, giving an incidence of 0.12 per 100,000 live births (95\% confidence interval 0.03-0.31). In addition, one pregnancy with an antenatal diagnosis underwent termination of pregnancy.

\section{Case Reports}

Case 1: Agenesis of the left lung was noted at the 20 week anomaly scan along with complex heart defects (complete atrioventricular septal defect with left atrial isomerism but normal spleen, transposed great arteries, right aortic arch and pulmonary stenosis). The baby was born in good condition at term. At three weeks of age she was readmitted with low oxygen saturations and underwent central shunt insertion (left subclavian artery to main pulmonary artery), which provided an adequate circulation until two years of age when she underwent corrective surgery.

Bronchoscopy revealed absence of complete tracheal rings, a blind ending stump to left main bronchus and moderate malacia of the right main bronchus. The patient has subsequently had multiple lower respiratory tract infections and received booster vaccinations after low specific titres were noted. Prophylactic antibiotics have also been prescribed. The patient is currently 7 years old and in mainstream schooling. Her mean oxygen saturations are $98 \%$ in air; spirometry at 6 years showed FEV1 53\% predicted with FVC 54\% predicted.

Case 2: Right lung aplasia was noted on MRI at 28 weeks while investigating abnormal cardiac views seen at the 20 week anomaly scan. The baby was born at term and did not require resuscitation. Postnatal echocardiograms confirmed coarctation of the aorta (repaired at 9 days) and an atrial septal defect (ASD; repaired at 3 months). The patient subsequently developed Wolff-Parkinson-White syndrome and underwent accessory pathway ablation at 6 years of age.

Previous reports have highlighted an association between lung aplasia and pulmonary hypertension; this seems to particularly occur with right-sided defects. [2] Our patient was treated for pulmonary hypertension following ASD repair, but treatment was discontinued after 3 months. The patient also had several urinary tract infections associated with Grade 1 vesicoureteral reflux but structurally normal kidneys. She is currently 10 years old and generally well, even taking part in charity running events. Spirometry at 8 years showed FEV1 of $55 \%$ predicted and FVC $56 \%$ predicted.

Case 3: Absence of the left lung was diagnosed at the 20 week anomaly scan. Subsequent investigations revealed a number of associated problems: segmental abnormalities of 
thoracic vertebrae 5 to 7 causing scoliosis, together with a dysplastic left thumb and bilateral grade 2 vesicoureteral reflux associated with recurrent urinary tract infections. This last problem is being managed with prophylactic antibiotics. Cardiac anatomy was normal other than an absent left pulmonary artery. Bronchoscopy at 15 months showed no evidence of bronchial stump on the left and normal right-sided anatomy.

The baby was born at term and required oxygen therapy shortly after birth. The subsequent clinical course has been dominated by recurrent lower respiratory tract infections. The patient was initially discharged home without oxygen but required continuous oxygen following a respiratory infection at 14 months: this was gradually weaned and stopped at age 2 years 10 months. The patient is currently 3 years old and doing well.

Case 4: This patient was born by spontaneous vaginal birth after an uncomplicated pregnancy with no antenatal concerns. She was initially well but was readmitted on day 2 of life with poor feeding and tachypnoea. Saturations were $86-88 \%$ in air. Chest $\mathrm{x}$-ray revealed absence of the left lung. She was discharged with home oxygen and made good progress other than several short admissions for respiratory tract infections. Echocardiogram showed a structurally normal heart and no evidence of pulmonary hypertension. Oxygen was gradually weaned and stopped after normal nocturnal saturation studies at 2 years of age.

\section{DISCUSSION}

Here we report the first accurate estimate of the incidence of congenital lung agenesis, which appears to be lower than previously estimated at 0.12 per 100,000 live births. Currently there are no known environmental associations with this condition and it is therefore presumed to be sporadic, suggesting the incidence will be relatively stable across comparable populations.

Medium-term outcomes in our patients have been more favourable than previous reports. All four patients have a good quality of life with no home oxygen requirement. It is important that these children are managed in a service which can provide early assessment of all associated anomalies. Specialist involvement should begin at diagnosis, which was antenatally in three of the four cases reported here. This allows personalised discussion of prognosis and expected course, and facilitates planning of future interventions. [5]

However, long-term outcome with modern management is still unknown, and a particular concern is the potential for the development of pulmonary hypertension in later life. We hope to follow-up these patients to clarify this issue.

Other than cardiac abnormalities, the two main clinical problems in our patients have been recurrent lower respiratory tract infections and vesico-ureteric reflux. Both require meticulous surveillance and judicious use of antibiotics. However, it is unclear whether the incidence of these co-morbidities is truly higher in patients with lung agenesis compared to the normal population, or whether the detection rate is higher because of closer medical surveillance.

\section{COMPETING INTERESTS - to amend with data from forms}


All authors have completed the ICMJE uniform disclosure form at www.icmje.org/coi_disclosure.pdf and declare: no support from any organisation for the submitted work; no financial relationships with any organisations that might have an interest in the submitted work in the previous three years; no other relationships or activities that could appear to have influenced the submitted work.

\section{CONTRIBUTORSHIP STATEMENT}

MT conceived and designed the work and wrote the manuscript; NR completed case summaries and statistical analysis, and wrote the manuscript; NM interrogated the NorCAS database; JR, MB and MM contributed to the manuscript.

\section{FUNDING}

MB is supported by a Medical Research Council Clinician Scientist Fellowship (MR/M008797/1)

\section{REFERENCES}

1 Krivchenya DU, Rudenko EO, Lysak SV, et al. Lung aplasia: anatomy, history, diagnosis and surgical management. Eur J Pediatr Surg 2007;17:244-50. doi:10.1055/s-2007965200

2 Muensterer O, Abellar R, Otterburn D, et al. Pulmonary Agenesis and Associated Pulmonary Hypertension: A Case Report and Review on Variability, Therapy, and Outcome. Eur J Pediatr Surg Rep 2015;3:33-9. doi:10.1055/s-0034-1395986

3 Russell BC, Whitecar $P$, Nitsche JF. Isolated unilateral pulmonary agenesis and other fetal thoracic anomalies. Obstet Gynecol Surv 2014;69:335-45. doi:10.1097/OGX.0000000000000070

4 Richmond S, Atkins J. A population-based study of the prenatal diagnosis of congenital malformation over 16 years. BJOG Int J Obstet Gynaecol 2005;112:1349-57. doi:10.1111/j.1471-0528.2005.00660.x

5 Hasegawa T, Oshima Y, Maruo A, et al. Pediatric cardiothoracic surgery in patients with unilateral pulmonary agenesis or aplasia. Ann Thorac Surg 2014;97:1652-8. doi:10.1016/j.athoracsur.2013.11.022 